\title{
Endometriosis, Ovarian Reserve and Live Birth Rate Following In Vitro Fertilization/ Intracytoplasmic Sperm Injection
}

\section{Endometriose, reserva ovariana e taxa de nascidos vivos após FIV/ICSI}

\author{
Marcela Alencar Coelho Neto ${ }^{1}$ Wellington de Paula Martins ${ }^{1}$ Caroline Mantovani da Luz ${ }^{1}$ \\ Bruna Talita Gazeto Melo Jianini ${ }^{1}$ Rui Alberto Ferriani ${ }^{1}$ Paula Andrea Navarro ${ }^{1}$
} 1 Department of Obstetrics and Gynecology, Faculdade de Medicina
de Ribeirão Preto, Universidade de São Paulo - USP, Ribeirão Preto,
SP, Brasil

Rev Bras Ginecol Obstet 2016;38:218-224.

\begin{abstract}
Address for correspondence Paula Andrea Navarro, MD, PhD, Departmento de Ginecologia e Obstetrícia, Faculdade de Medicina de Ribeirão Preto, Hospital das Clínicas de Ribeirão Preto, Centro de Reprodução Humana, Universidade de São Paulo, Avenida Bandeirantes, 3.900, 80 andar, Ribeirão Preto, caixa postal: 14048900, Ribeirão Preto, SP, Brazil (e-mail: pnavarro@fmrp.usp.br).
\end{abstract}

\begin{abstract}
Keywords

- endometriosis

- ovarian reserve

- assisted reproduction

- IVF

$-\mid \mathrm{ICSI}$
\end{abstract}

Purpose To evaluate whether women with endometriosis have different ovarian reserves and reproductive outcomes when compared with women without this diagnosis undergoing in vitro fertilization/intracytoplasmic sperm injection (IVF/ ICSI), and to compare the reproductive outcomes between women with and without the diagnosis considering the ovarian reserve assessed by antral follicle count (AFC). Methods This retrospective cohort study evaluated all women who underwent IVF/ ICSI in a university hospital in Brazil between January 2011 and December 2012. All patients were followed up until a negative pregnancy test or until the end of the pregnancy. The primary outcomes assessed were number of retrieved oocytes and live birth. Women were divided into two groups according to the diagnosis of endometriosis, and each group was divided again into a group that had AFC $\leq 6$ (poor ovarian reserve) and another that had AFC $\geq 7$ (normal ovarian reserve). Continuous variables with normal distribution were compared using unpaired $t$-test, and those without normal distribution, using Mann-Whitney test. Binary data were compared using either Fisher's exact test or Chi-square $\left(\chi^{2}\right)$ test. The significance level was set as $p<0.05$. Results 787 women underwent IVF/ICSI (241 of which had endometriosis). Although the mean age has been similar between women with and without the diagnosis of endometriosis ( $33.8 \pm 4$ versus $33.7 \pm 4.4$ years, respectively), poor ovarian reserves were much more common in women with endometriosis (39.8 versus $22.7 \%$ ). The chance of achieving live birth was similar between women with the diagnosis of endometriosis and those without it (19.1 versus $22.5 \%)$, and also when considering only women with a poor ovarian reserve (9.4 versus $8.9 \%$ ) and only those with a normal ovarian reserve (25.5 versus $26.5 \%$ ). received

January 5, 2016

accepted

March 30, 2016

published online

May 19, 2016
Copyright $\odot 2016$ by Thieme Publicações License terms

Ltda, Rio de Janeiro, Brazil
(๑) $\Theta \circledast$ 


\section{Resumo}

Palavras-chave
- endometriose
- reserva ovariana
- reprodução
assistida
- fertilização in
virto
- ICSI

Conclusions Women diagnosed with endometriosis are more likely to have a poor ovarian reserve; however, their chance of conceiving by IVF/ICSI is similar to the one observed in patients without endometriosis and with a comparable ovarian reserve.

Objetivo Avaliar se mulheres com endometriose possuem diferenças quanto a reserva ovariana (RO) e a resultados de reprodução assistida quando comparadas a mulheres sem este diagnóstico submetidas IVF/ICSI (in vitro fertilization/intracytoplasmic sperm injection), e comparar resultados reprodutivos entre mulheres com e sem o diagnóstico, considerando a RO obtida pela contagem de folículos antrais (CFA).

Métodos Este estudo de coorte retrospectivo avaliou todas as mulheres submetidas à FIV/ICSI em uma universidade do Brasil nos anos de 2011 e 2012. Todas as pacientes foram seguidas até um teste negativo de gravidez ou até o final da gestação. Os desfechos primários analisados foram o número do oócitos captados e nascidos vivos. As mulheres foram divididas em 2 grupos de acordo com o diagnóstico de endometriose e subdivididas de acordo com a CFA $\leq 6$ (baixa RO) e $\geq 7$ (RO normal). As variáveis contínuas com distribuição normal foram comparadas pelo teste $t$ não pareado e sem distribuição normal pelo teste de Mann-Whitney. Os dados binários foram comparados por ambos os testes Qui-quadrado $\left(\chi^{2}\right)$ e exato de Fisher. O nível de significância foi definido como $p<0,05$.

Resultados 787 mulheres foram submetidas a IVF/ICSI (241 com endometriose). Embora a média de idade tenha sido similar entre as mulheres com e sem o diagnóstico de endometriose (33,8 \pm 4 versus 33,7 \pm 4.4 anos, respectivamente), a baixa RO é muito mais comum em mulheres com endometriose ( 39,8 versus $22,7 \%$ ). A chance de obter um nascido vivo foi similar entre as mulheres com e sem endometriose $(19,1$ versus $22,5 \%)$, e também quando consideradas apenas as mulheres com baixa RO $(9,4$ versus $8,9 \%$ ), e apenas com RO normal ( 25,5 versus $26,5 \%$ ).

Conclusões Mulheres diagnosticadas com endometriose são mais susceptíveis a ter baixa RO; no entanto, suas chances de conceber por IVF/ICSI são similares às observadas em pacientes sem endometriose e com uma RO comparável.

\section{Introduction}

Subfertility is the inability of achieving a pregnancy after one year of regular, unprotected intercourse. ${ }^{1}$ Approximately $15 \%$ of women in reproductive age are considered subfertile. ${ }^{2-4}$ Assisted Reproduction Technologies (ARTs), particularly in vitro fertilization (IVF) and intracytoplasmic sperm injection (ICSI), are frequently used to help subfertile couples to conceive.

Endometriosis is one of the most cited causes of subfertility, being much more common in subfertile (25-40\%) than in fertile woman $(0.5-5.0 \%){ }^{5}$ This disease is defined by the presence of endometrial tissue outside the uterine cavity, and the mechanisms through which endometriosis impairs fertility are still unclear. ${ }^{6}$ Pelvic adhesions and distortion of pelvic anatomy are associated with subfertility, and are frequently observed in moderate and severe cases of endometriosis. ${ }^{7}$ However, the mechanism underlying minimal or mild endometriosis related to subfertility is unclear, and may be related to impairment of oocyte development, embryogenesis and implantation. ${ }^{8-10}$ The ideal fertility treatment for women with endometriosis is not well established yet, but ART treatments are responsible for the highest success rates. ${ }^{11,12}$

The relationship between the presence and severity of endometriosis and the reproductive outcomes in women undergoing ART are controversial. ${ }^{13-15}$ The first meta-analysis evaluating this issue found that endometriosis reduces the chances of achieving pregnancy in IVF/ICSI cycles, and that patients with moderate to severe endometriosis (III/IV) have even worse results. ${ }^{14}$ The second review observed that only severe endometriosis impairs implantation and clinical pregnancy rates when compared with women without the disease. ${ }^{15}$ However, the latest and largest published review found moderate quality evidence suggesting that endometriosis does not affect the chance of achieving clinical pregnancy and live birth when considering only the fresh transfer cycle. ${ }^{13}$ Furthermore, the same review suggests that patients with endometriosis have a reduced ovarian reserve, once fewer oocytes are retrieved from women in this group. Since a poor ovarian reserve is associated with poor reproductive outcomes, ${ }^{16,17}$ it is possible that the differences in the ovarian reserve, and not endometriosis itself, might be the cause of the heterogeneity observed in the studies published so far. ${ }^{13}$ 
Thus, the objective of this study was to evaluate whether women with endometriosis have different ovarian reserves and reproductive outcomes when compared with women without this diagnosis undergoing IVF/ICSI. We also compared the reproductive outcomes between women with and without the diagnosis of endometriosis considering the ovarian reserve assessed by antral follicle count (AFC) evaluated before the beginning of the controlled ovarian stimulation (COS) for FIV/ICSI.

\section{Methods}

This retrospective cohort study included all women who underwent IVF/ICSI at the fertility clinic of the university hospital of the Ribeirão Preto Medical School, Universidade de São Paulo, Brazil, between January 2011 and December 2012. All data were obtained from medical records by one author. The study protocol was approved by the Institutional Review Board, which waived the requirement for additional informed consent due to the retrospective nature of the study.

Women were considered eligible if they had undergone IVF/ICSI between January 2011 and December 2012 at our fertility clinic. If women had undergone more than one cycle of COS during this period, only the data from the first cycle with planned embryo transfer were included. All women were followed up until a negative pregnancy test or the end of a pregnancy.

In or der to divide the women into groups, we assessed the diagnosis of endometriosis made by laparoscopy and/or ultrasound and the baseline AFC, performed before the beginning of the treatment by transvaginal ultrasound using a Voluson E8 Expert (GE Healthcare Austria GmbH \& Co OG, Zipf, Austria) operated by one of four well-trained sonologists. Ultrasound is considered the first-line imaging method for women with suspected pelvic endometriosis: although it fails to identify superficial endometriosis, the method has an excellent diagnostic accuracy for endometrioma and deep infiltrating endometriosis. ${ }^{18-23}$

The unit of analysis of all parameters was the number of women. The primary endpoints of this study were the number of retrieved oocytes and the rates of live birth. The following parameters were assessed: clinical pregnancy, presence of surplus frozen embryos, occurrence of embryo transfer and oocyte retrieval, cancellation of the cycle due to poor response, number of oocytes retrieved, baseline AFC, body mass index (BMI), age, and the protocol used for COS. In cases in which women did not undergo oocyte retrieval and embryo transfer, we considered that the number of both oocytes retrieved and embryos transferred was zero; by doing that, all women were included in the analysis. Furthermore, for $5.7 \%$ of women, AFC data was missing; so, for subgrouping purposes, we considered that those patients had an AFC $\geq 7$, and grouped them within the largest subgroup. All variables were obtained from the medical records.

\section{Statistical Analysis}

First, women were divided into two groups: those that had and those that did not have the diagnosis of endometriosis.
For a second analysis, women were further stratified into two subgroups: $\mathrm{AFC} \leq 6$ and $\mathrm{AFC} \geq 7$. This latter analysis was performed to separate women with expected poor ovarian response according to the AFC cutoff values suggested in the Bologna criteria. ${ }^{24}$ The study size was determined by the primary outcome number of oocytes retrieved. Considering a difference of one oocyte retrieved as being clinically relevant, ${ }^{25}$ a standard deviation (SD) of 4.5 oocytes retrieved at our service, ${ }^{26}$ a proportion of women with endometriosis to be close to $30 \%,{ }^{13}$ significance level $=p<0.05$, we would need 760 women to have a power of $80 \%$ to detect this difference. A mean of 40 women are submitted to treatment cycle per month in our clinic. Considering that part of these women repeat the treatment in a short period of time, we calculated that we would need to retrieve data from 24 months in order to examine the estimated sample size. With that sample size, the power to detect an absolute $5 \%$ decrease in live birth rates was $40 \%$, and of detecting a decrease of $10 \%$ in live birth rates was $90 \%$.

The normality of distribution of continuous variables was analyzed using the Kolmogorov-Smirnov test. Continuous variables with normal distribution were summarized as mean \pm SD and compared between the two groups using unpaired $t$-test. Continuous variables without normal distribution were summarized as median (interquartile range) and comparisons were made between the two groups using Mann-Whitney test. Binary data were presented as ratio and proportion and compared between the two groups using either Fisher's exact test or Chi-square $\left(\chi^{2}\right)$ test. The level of significance was defined as $p<0.05$. We also calculated the relative risk (RR), the mean difference (MD) and their 95\% confidence interval $(\mathrm{CI})$ for the studied outcomes. All statistical analysis were performed using SPSS (version 18.0, SPSS Inc., Chicago, IL, USA).

\section{Results}

During the study period, 787 women underwent IVF/ICSI in our clinic; data were analyzed from all 787 cycles. All women were followed until a negative pregnancy test or the end of the pregnancy, with reproductive outcomes analyzed in all cases.

In the analysis of the 787 women included, 241 (30.6\%) were diagnosed with endometriosis. The diagnosis of endometriosis was performed by ultrasound in only 30 participants (12.4\%); by laparoscopy in 182 of them (75.5\%); and by both laparoscopy and ultrasound in 29 subjects (12.0\%). Considering the 546 women without the diagnosis of endometriosis, 151 (26.7\%) underwent laparoscopy with negative findings for endometriosis, and 395 women presented no clinical or ultrasonographic suspicion of endometriosis, and, therefore, did not undergo laparoscopy. In the subgroup with a poor ovarian reserve (AFC $\leq 6), 220$ women were included, and of these, 96 (43.6\%) had endometriosis. Among these women, the diagnosis of endometriosis was performed by ultrasound in only 12 (12.5\%) of them; by laparoscopy in 70 (72.9\%) of them; and by both laparoscopy and ultrasound in 14 women (14.6\%). In the subgroup without a poor ovarian reserve (AFC $\geq 7$ ), 567 women were included, and of these, 
145 (25.6\%) had endometriosis. Among these patients, the diagnosis of endometriosis was performed by ultrasound in only $18(12.4 \%)$ of them; by laparoscopy in $112(77.2 \%)$ of them; and by both laparoscopy and ultrasound in 15 women (10.3\%).

Regarding the type of COS, 68.1\% (536 women) used gonadotropin-releasing hormone $(\mathrm{GnRH})$ antagonist protocol with standard exogenous gonadotropins (150300IU/day); 21.1\% (166 women) used GnRH agonist protocol with standard exogenous gonadotropins; and $10.8 \%$ (85 women) used clomiphene citrate with low-dose exogenous gonadotropins ( $<150 \mathrm{UI} /$ day). For final follicular maturation, $93.9 \%$ (739 women) used urinary or recombinant human chorionic gonadotropin (hCG) for triggering, while $6.1 \%$ (48 women) used GnRH agonist. Oocytes were retrieved 34 to 36 hours after the triggering. The distribution of COS protocols between the groups is described in -Tables 1, 2 and 3.

When considering all women, no significant difference was observed between the two groups regarding age, and BMI was slightly lower in the endometriosis group. Parameters of ovarian reserve and response to the treatment were worse among women with endometriosis: they had lower AFC, fewer oocytes retrieved, more cycles cancelled due to poor response, fewer women underwent oocyte retrieval/ embryo transfer, and fewer had surplus frozen embryos.
Notwithstanding, there was no evidence of difference on live birth rates and clinical pregnancy (-Table $\mathbf{1}$ ).

We also performed a subgroup analysis, stratifying women into two subgroups: those with a poor ovarian reserve $(\mathrm{AFC} \leq 6)$ and those without a poor ovarian reserve (AFC $\geq 7$ ). The proportion of women with AFC $\leq 6$ was significantly higher in participants with endometriosis: 39.8 versus $22.7 \%$ (-Table 1). Among women with a poor ovarian reserve, we observed that those with endometriosis were significantly younger, with no difference on BMI. Unlike the previous analysis, there was no difference in the parameters related to the response of the treatment between women with and without endometriosis: number of oocytes retrieved, number of cycles cancelled due to poor response, proportion of women undergoing oocyte retrieval and embryo transfer, proportion of women with surplus frozen embryos, live birth, and clinical pregnancy. Notwithstanding, AFC was lower in the endometriosis group ( - Table 2 ). In the subgroup of women without a poor ovarian reserve (AFC $\geq 7$ ), we did not observe any difference in age, but women with endometriosis had a lower BMI. Women with endometriosis presented lower AFC and less oocytes retrieved, while no difference was observed in the number of cycles cancelled due to poor response, the proportion of women undergoing oocyte retrieval and embryo transfer, the proportion of women with surplus frozen embryos, live birth rates, and clinical pregnancy.

Table 1 Evaluated parameters of all women undergoing IVF/ICSI during the study period divided based on the diagnosis of endometriosis

\begin{tabular}{|c|c|c|c|c|c|c|c|}
\hline \multirow[t]{2}{*}{ Parameter } & \multicolumn{3}{|c|}{ Endometriosis } & \multicolumn{3}{|l|}{ Control } & \multirow[b]{2}{*}{$p^{*}$} \\
\hline & Mean & SD & $\mathrm{N}$ & Mean & SD & $\mathrm{N}$ & \\
\hline Age & 34.4 & 4.3 & 241 & 34.5 & 4.5 & 546 & 0.7 \\
\hline \multirow[t]{2}{*}{ BMI } & 24.2 & 4.0 & 225 & 25.1 & 4.2 & 525 & 0.01 \\
\hline & Median & IQR & $\mathrm{N}$ & Median & IQR & $\mathrm{N}$ & $p^{* *}$ \\
\hline AFC & 8.0 & $4-13$ & 233 & 12.0 & $7-20$ & 509 & $<0.001$ \\
\hline \multirow[t]{2}{*}{ Number of oocytes retrieved } & 4.0 & $2-8$ & 241 & 6.0 & $3-10$ & 546 & $<0.001$ \\
\hline & $\%$ & $\mathbf{n}$ & $\mathrm{N}$ & $\%$ & $\mathbf{n}$ & $\mathrm{N}$ & $p^{* * *}$ \\
\hline Proportion of women with AFC $\leq 6$ & $39.8 \%$ & 96 & 241 & $22.7 \%$ & 124 & 546 & $<0.001$ \\
\hline Cycle cancelled - poor response & $6.2 \%$ & 15 & 241 & $2.7 \%$ & 15 & 546 & 0.03 \\
\hline Underwent OR & $92.5 \%$ & 223 & 241 & $97.1 \%$ & 530 & 546 & 0.007 \\
\hline Underwent ET & $77.2 \%$ & 186 & 241 & $83.2 \%$ & 454 & 546 & 0.06 \\
\hline Women with surplus embryos & $32.0 \%$ & 77 & 241 & $42.1 \%$ & 230 & 546 & 0.007 \\
\hline Clinical pregnancy & $22.4 \%$ & 54 & 241 & $27.5 \%$ & 150 & 546 & 0.2 \\
\hline Live birth & $19.1 \%$ & 46 & 241 & $22.5 \%$ & 123 & 546 & 0.3 \\
\hline \multicolumn{8}{|l|}{$\cos$} \\
\hline $\mathrm{FSH}+\mathrm{Ant}$ & $63.1 \%$ & 152 & 241 & $72.7 \%$ & 397 & 546 & \multirow[t]{3}{*}{0.01} \\
\hline $\mathrm{FSH}+\mathrm{Ago}$ & $22.8 \%$ & 55 & 241 & $19.4 \%$ & 106 & 546 & \\
\hline $\mathrm{CC}+\mathrm{FSH}+\mathrm{Ant}$ & $14.1 \%$ & 34 & 241 & $7.9 \%$ & 43 & 546 & \\
\hline
\end{tabular}

Abbreviations: AFC, antral follicle count; Ago, agonist protocol; Ant, antagonist protocol; BMI, body mass index; CC, clomiphene citrate; COS, controlled ovarian stimulation; ET, embryo transfer; FSH, follicle-stimulating hormone; IQR, interquartile range; n, number of events; $\mathrm{N}$, number of woman analyzed; OR, oocyte retrieval; SD, standard deviation.

Notes: ${ }^{*} p$ values calculated by unpaired $t$-test; ${ }^{* *} p$ values calculated by Mann-Whitney test; ${ }^{* * *} p$ values calculated by Fisher exact test or Chi-square $\left(\chi^{2}\right)$ test. 
Table 2 Evaluated parameters of women undergoing IVF/ICSI during the study period with a poor ovarian reserve (AFC $\leq 6$ ) divided based on the diagnosis of endometriosis

\begin{tabular}{|l|l|l|l|l|l|l|l|}
\hline Parameter & \multicolumn{3}{|l|}{ Endometriosis } & \multicolumn{2}{l|}{ Control } & \\
\hline & Mean & SD & N & Mean & SD & N & $p^{*}$ \\
\hline Age & 35.2 & 4.5 & 96 & 37.3 & 3.6 & 124 & $<0.001$ \\
\hline BMI & 24.2 & 4.4 & 90 & 24.4 & 4.2 & 119 & 0.8 \\
\hline & Median & IQR & $\mathbf{N}$ & Median & IQR & $\mathbf{N}$ & $p^{* *}$ \\
\hline AFC & 3.5 & $3.0-5.0$ & 96 & 5.0 & $3.3-6.0$ & 124 & $<0.001$ \\
\hline Number of oocytes retrieved & 2.00 & $0.3-4.8$ & 96 & 3.0 & $1.0-5.0$ & 124 & 0.2 \\
\hline & $\%$ & $\mathbf{n}$ & $\mathbf{N}$ & $\%$ & $\mathbf{n}$ & $\mathbf{N}$ & $p^{* * *}$ \\
\hline Cycle cancelled - poor response & $11.5 \%$ & 11 & 96 & $7.3 \%$ & 9 & 124 & 0.4 \\
\hline Underwent OR & $87.5 \%$ & 84 & 96 & $92.7 \%$ & 115 & 124 & 0.3 \\
\hline Underwent ET & $65.6 \%$ & 63 & 96 & $66.1 \%$ & 82 & 124 & 1.0 \\
\hline Women with surplus embryos & $14.6 \%$ & 14 & 96 & $14.5 \%$ & 18 & 124 & 1.0 \\
\hline Clinical pregnancy & $11.5 \%$ & 11 & 96 & $13.7 \%$ & 17 & 124 & 0.7 \\
\hline Live birth & $9.4 \%$ & 9 & 96 & $8.9 \%$ & 11 & 124 & 1.0 \\
\hline COS & & & & & & & \\
\hline FSH + Ant & $52.1 \%$ & 50 & 96 & $69.4 \%$ & 86 & 124 & 0.03 \\
\hline FSH + Ago & $17.7 \%$ & 17 & 96 & $10.5 \%$ & 13 & 124 & \\
\hline CC + FSH + Ant & $30.2 \%$ & 29 & 96 & $20.2 \%$ & 25 & 124 & \\
\hline
\end{tabular}

Abbreviations: AFC, antral follicle count; Ago, agonist protocol; Ant, antagonist protocol; BMI, body mass index; CC, clomiphene citrate; COS, controlled ovarian stimulation; ET, embryo transfer; FSH, follicle-stimulating hormone; IQR, interquartile range; $n$, number of events; $\mathrm{N}$, number of woman analyzed; OR, oocyte retrieval; SD, standard deviation.

Notes: ${ }^{*} p$ values calculated by unpaired $t$-test; ${ }^{* *} p$ values calculated by Mann-Whitney test; ${ }^{* * *} p$ values calculated by Fisher exact test or Chi-square $\left(\chi^{2}\right)$ test.

Table 3 Evaluated parameters of women undergoing IVF/ICSI during the study period without a poor ovarian reserve (AFC $\geq 7$ ) divided based on the diagnosis of endometriosis

\begin{tabular}{|c|c|c|c|c|c|c|c|}
\hline \multirow[t]{2}{*}{ Parameter } & \multicolumn{3}{|c|}{ Endometriosis } & \multicolumn{3}{|l|}{ Control } & \multirow[b]{2}{*}{$p^{*}$} \\
\hline & Mean & SD & $\mathrm{N}$ & Mean & SD & $\mathrm{N}$ & \\
\hline Age & 33.8 & 4.0 & 145 & 33.7 & 4.4 & 422 & 0.7 \\
\hline \multirow[t]{2}{*}{ BMI } & 24.2 & 3.8 & 135 & 25.4 & 4.2 & 406 & 0.01 \\
\hline & Median & IQR & $\mathrm{N}$ & Median & IQR & $\mathbf{N}$ & $p^{* *}$ \\
\hline AFC & 12.0 & $9.0-17.5$ & 137 & 15.0 & $10.0-22.0$ & 385 & $<0.001$ \\
\hline \multirow[t]{2}{*}{ Number of oocytes retrieved } & 6.0 & $3.0-10.0$ & 145 & 7.0 & $4.75-11.0$ & 422 & 0.02 \\
\hline & $\%$ & $\mathrm{n}$ & $\mathrm{N}$ & $\%$ & $\mathrm{n}$ & $\mathrm{N}$ & $p^{* * *}$ \\
\hline Cycle cancelled - poor response & $2.8 \%$ & 4 & 145 & $1.4 \%$ & 6 & 422 & 0.3 \\
\hline Underwent OR & $95.9 \%$ & 139 & 145 & $98.3 \%$ & 415 & 422 & 0.1 \\
\hline Underwent ET & $84.8 \%$ & 123 & 145 & $88.2 \%$ & 372 & 422 & 0.3 \\
\hline Women with surplus embryos & $43.4 \%$ & 63 & 145 & $50.2 \%$ & 212 & 422 & 0.2 \\
\hline Clinical pregnancy & $29.7 \%$ & 43 & 145 & $31.5 \%$ & 133 & 422 & 0.8 \\
\hline Live birth & $25.5 \%$ & 37 & 145 & $26.5 \%$ & 112 & 422 & 0.9 \\
\hline \multicolumn{8}{|l|}{$\cos$} \\
\hline $\mathrm{FSH}+\mathrm{Ant}$ & $70.3 \%$ & 102 & 145 & $73.7 \%$ & 311 & 422 & \multirow[t]{3}{*}{0.6} \\
\hline $\mathrm{FSH}+\mathrm{Ago}$ & $26.2 \%$ & 38 & 145 & $22.0 \%$ & 93 & 422 & \\
\hline $\mathrm{CC}+\mathrm{FSH}+\mathrm{Ant}$ & $3.4 \%$ & 5 & 145 & $4.3 \%$ & 18 & 422 & \\
\hline
\end{tabular}

Abbreviations: AFC, antral follicle count; Ago, agonist protocol; Ant, antagonist protocol; BMI, body mass index; CC, clomiphene citrate; COS, controlled ovarian stimulation; ET, embryo transfer; FSH, follicle-stimulating hormone; IQR, interquartile range; n, number of events; N, number of woman analyzed; OR, oocyte retrieval; SD, standard deviation.

Notes: ${ }^{*}$ values calculated by unpaired $t$-test; ${ }^{* *}$ P values calculated by Mann-Whitney test; ${ }^{* * *}$ P values calculated by Fisher exact test or Chi-square $\left(\chi^{2}\right)$ test. 


\section{Discussion}

This study showed that although the mean age was similar between women with and without the diagnosis of endometriosis, the proportion of participants with a poor ovarian reserve was significantly higher among young women diagnosed with endometriosis. Furthermore, women with endometriosis had fewer oocytes retrieved. Among women with a decreased functional ovarian reserve, those with endometriosis were significantly younger. Although the live birth rate might be considered slightly lower in women with endometriosis (19.1 versus $22.5 \%$ ) without significant difference, it was very similar when considering only women with a low ovarian reserve (9.4 versus $8.9 \%$ ) or a normal ovarian reserve (25.5 versus $26.5 \%$ ).

A recent review found moderate quality evidence suggesting that endometriosis does not affect the chance of achieving live birth and clinical pregnancy when considering the first embryo transfer, ${ }^{13}$ which is in agreement with our results. Additionally, the same review observed that women with endometriosis have fewer oocytes retrieved, suggesting a reduced ovarian reserve, which is also in agreement with the findings of the present study. However, some of the studies published so far have observed significantly worse results in women with endometriosis. ${ }^{27-29}$ We believe that the difference among studies might be explained by the reduced ovarian reserve associated with endometriosis, as this reduction is related to poor assisted reproduction outcomes. ${ }^{16} \mathrm{~A}$ recent and large observational study ${ }^{16}$ examined data from more than 140,000 women with a single diagnosis of infertility; those with more than one cause were excluded from the analysis. Since this study considered that a reduced ovarian reserve was one of the causes of infertility, women with both endometriosis and a reduced ovarian reserve were not analyzed. This interesting study observed that couples with only endometriosis have live birth rates (43.0\%) very similar to those observed in couples with only male factor (42.7\%), with only ovulatory dysfunction (44.1\%), and those with unexplained infertility (42\%); however, all of these rates were much higher than those observed in couples in which the woman had a reduced ovarian reserve and no other cause of subfertility was detected (18.9\%). ${ }^{16}$ Since women with endometriosis are more likely to have a reduced ovarian reserve, there is a reasonable chance to observe worse reproductive outcomes in women with endometriosis by including all women without controlling for this confounding factor.

Another very important observation is that among women with a decreased ovarian reserve, those with endometriosis are younger. Since age and ovarian reserve are the most important factors for the treatment's success, ${ }^{16,30}$ when balancing risks and benefits, women with suspected endometriosis might be at an increased risk of facing a reduced ovarian reserve at an earlier age. The costs and psychological harms of an early fertility treatment should be weighed against the risk of a reduced ovarian reserve and, therefore, worse reproductive outcomes for both natural conception and subsequent assisted reproductive techniques, caused by the delay in treatment on behalf of a more precise diagnosis or intervention for endometriosis. Another interesting point is that women with endometriosis are more likely to have endometrioma, which interferes more with the AFC than with the number of oocytes retrieved and the chances of conceiving. ${ }^{30,31}$

As this is a retrospective study, we had to rely on data from our medical records. Antral follicle count data was missing in $3 \%$ of the women with endometriosis and in $6 \%$ of the controls; therefore, for the subgroup analysis we assumed that all missing AFC were $\geq 7$. This source of bias could be overcome by a prospective study. Another issue is that not all women underwent a laparoscopy for the diagnosis of endometriosis. If on one hand it could be a source of bias because some participants lacked a precise diagnostic evaluation, on the other hand it improves generalization, since not every woman undergoes laparoscopy previously to IVF/ICSI.

The generalization of the findings described is limited because this is a retrospective observational study; however, it should encourage the development of new research evaluating reproductive outcomes in patients diagnosed with endometriosis taking into account their ovarian reserve.

In conclusion, among women undergoing IVF/ICSI, those with endometriosis are more likely to have a poor ovarian reserve. However, women diagnosed with endometriosis have similar chances of conceiving when compared with other women with a similar ovarian reserve.

\section{Acknowledgments}

The authors are grateful to Universidade de São Paulo (USP), the Foundation for Support in Teaching, Research and Assistance (Faepa), and the Brazilian National Council for Research and Development (CNPq) for their financial support.

\section{References}

1 Gnoth C, Godehardt E, Frank-Herrmann P, Friol K, Tigges J, Freundl G. Definition and prevalence of subfertility and infertility. Hum Reprod 2005;20(5):1144-1147

2 Bushnik T, Cook JL, Yuzpe AA, Tough S, Collins J. Estimating the prevalence of infertility in Canada. Hum Reprod 2012;27(3): 738-746

3 Thoma ME, McLain AC, Louis JF, et al. Prevalence of infertility in the United States as estimated by the current duration approach and a traditional constructed approach. Fertil Steril 2013;99(5): 1324-1331.e1

4 Bhattacharya S, Porter M, Amalraj E, et al. The epidemiology of infertility in the North East of Scotland. Hum Reprod 2009;24(12):3096-3107

5 Ozkan S, Murk W, Arici A. Endometriosis and infertility: epidemiology and evidence-based treatments. Ann N Y Acad Sci 2008; 1127:92-100

6 Ahn SH, Monsanto SP, Miller C, Singh SS, Thomas R, Tayade C. Pathophysiology and immune dysfunction in endometriosis. Biomed Res Int 2015;2015:795976

7 Practice Committee of the American Society for Reproductive Medicine. Endometriosis and infertility: a committee opinion. Fertil Steril 2012;98(3):591-598

8 Gupta S, Goldberg JM, Aziz N, Goldberg E, Krajcir N, Agarwal A. Pathogenic mechanisms in endometriosis-associated infertility. Fertil Steril 2008;90(2):247-257 
9 Barcelos ID, Vieira RC, Ferreira EM, Martins WP, Ferriani RA, Navarro PA. Comparative analysis of the spindle and chromosome configurations of in vitro-matured oocytes from patients with endometriosis and from control subjects: a pilot study. Fertil Steril 2009;92(5):1749-1752

10 Da Broi MG, Malvezzi H, Paz CC, Ferriani RA, Navarro PA. Follicular fluid from infertile women with mild endometriosis may compromise the meiotic spindles of bovine metaphase II oocytes. Hum Reprod 2014;29(2):315-323

11 de Ziegler D, Borghese B, Chapron C. Endometriosis and infertility: pathophysiology and management. Lancet 2010;376(9742): 730-738

12 Dunselman GA, Vermeulen N, Becker C, et al; European Society of Human Reproduction and Embryology. ESHRE guideline: management of women with endometriosis. Hum Reprod 2014;29(3): 400-412

13 Barbosa MA, Teixeira DM, Navarro PA, Ferriani RA, Nastri CO, Martins WP. Impact of endometriosis and its staging on assisted reproduction outcome: systematic review and meta-analysis. Ultrasound Obstet Gynecol 2014;44(3):261-278

14 Barnhart K, Dunsmoor-Su R, Coutifaris C. Effect of endometriosis on in vitro fertilization. Fertil Steril 2002;77(6):1148-1155

15 Harb HM, Gallos ID, Chu J, Harb M, Coomarasamy A. The effect of endometriosis on in vitro fertilisation outcome: a systematic review and meta-analysis. BJOG 2013;120(11):1308-1320

16 Stern JE, Brown MB, Wantman E, Kalra SK, Luke B. Live birth rates and birth outcomes by diagnosis using linked cycles from the SART CORS database. J Assist Reprod Genet 2013;30(11): $1445-1450$

17 Ruth KS, Beaumont RN, Tyrrell J, et al. Genetic evidence that lower circulating FSH levels lengthen menstrual cycle, increase age at menopause and impact female reproductive health. Hum Reprod 2016;31(2):473-481

18 Exacoustos C, Manganaro L, Zupi E. Imaging for the evaluation of endometriosis and adenomyosis. Best Pract Res Clin Obstet Gynaecol 2014;28(5):655-681

19 Guerriero S, Ajossa S, Minguez JA, et al. Accuracy of transvaginal ultrasound for diagnosis of deep endometriosis in uterosacral ligaments, rectovaginal septum, vagina and bladder: systematic review and meta-analysis. Ultrasound Obstet Gynecol 2015; 46(5):534-545

20 Guerriero S, Ajossa S, Orozco R, et al. Accuracy of transvaginal ultrasound for diagnosis of deep endometriosis in the rectosig- moid: systematic review and meta-analysis. Ultrasound Obstet Gynecol 2016;47(3):281-289

21 Nisenblat V, Bossuyt PM, Farquhar C, Johnson N, Hull ML. Imaging modalities for the non-invasive diagnosis of endometriosis. Cochrane Database Syst Rev 2016;2:CD009591

22 Noventa M, Saccardi C, Litta P, et al. Ultrasound techniques in the diagnosis of deep pelvic endometriosis: algorithm based on a systematic review and meta-analysis. Fertil Steril 2015;104(2): 366-83.e2

23 Sotiriadis A, Papatheodorou SI, Martins WP. Synthesizing Evidence from Diagnostic Accuracy TEsts: the SEDATE guideline. Ultrasound Obstet Gynecol 2016;47(3):386-395

24 Ferraretti AP, La Marca A, Fauser BC, Tarlatzis B, Nargund G, Gianaroli L; ESHRE working group on Poor Ovarian Response Definition. ESHRE consensus on the definition of 'poor response' to ovarian stimulation for in vitro fertilization: the Bologna criteria. Hum Reprod 2011;26(7):1616-1624

25 Figueiredo JB, Nastri CO, Vieira AD, Martins WP. Clomiphene combined with gonadotropins and GnRH antagonist versus conventional controlled ovarian hyperstimulation without clomiphene in women undergoing assisted reproductive techniques: systematic review and meta-analysis. Arch Gynecol Obstet 2013; 287(4):779-790

26 Picinato MC, Martins WP, Giorgenon RC, et al. The impact of examining the meiotic spindle by polarization microscopy on assisted reproduction outcomes. Fertil Steril 2014;101(2):379-384

27 Lin XN, Wei ML, Tong XM, et al. Outcome of in vitro fertilization in endometriosis-associated infertility: a 5-year database cohort study. Chin Med J (Engl) 2012;125(15):2688-2693

28 Simón C, Gutiérrez A, Vidal A, et al. Outcome of patients with endometriosis in assisted reproduction: results from in-vitro fertilization and oocyte donation. Hum Reprod 1994;9(4):725-729

29 Surrey ES. Endometriosis-related infertility: the role of the assisted reproductive technologies. Biomed Res Int 2015; 2015:482959

30 Coelho Neto MA, Martins WP, Lima ML, et al. Ovarian response is a better predictor of clinical pregnancy rate following embryo transfer than is thin endometrium or presence of an endometrioma. Ultrasound Obstet Gynecol 2015;46(4):501-505

31 Lima ML, Martins WP, Coelho Neto MA, Nastri CO, Ferriani RA, Navarro PA. Assessment of ovarian reserve by antral follicle count in ovaries with endometrioma. Ultrasound Obstet Gynecol 2015; 46(2):239-242 